\title{
DO I STILL NEED MY BRAIN? EVOLUTION OF NAÏVE MIND-BRAIN CONCEPTIONS FROM CHILDHOOD TO ADULTHOOD
}

\author{
Sandrine Rossi ${ }^{1}$, Pauline Allix ${ }^{1}$, Céline Lanoë ${ }^{1}, \&$ Amélie Lubin ${ }^{2}$ \\ ${ }^{1}$ Laboratoire de Psychologie Caen Normandie EA 7452, Université de Caen Normandie (France) \\ ${ }^{2}$ Laboratoire de Psychologie Caen Normandie EA 7452, Université Paris Descartes (France)
}

\begin{abstract}
Given that metacognitive knowledge promotes students' academic learning, it seems important to better understand how the mind-brain conceptions change with age. We recruited 34 children aged 5/6 years, 34 children aged 7/8 years, 33 pre-adolescents aged 10/12 years, 30 adolescents aged 14/16 years, and 33 adults aged 21 to 35 years. We used the Mind-Brain Questionnaire (Rossi et al., 2015). Each participant was asked to indicate what a character needs in order to perform different actions by using response cards (brain, mind, hand, heart, eye, mouth). We conducted analyses for four response category 1- Mind and Brain response cards; 2- Brain without Mind response cards; 3- Mind without Brain response cards; 4- Neither Mind nor Brain response cards. The results reveal that participants over 10 years of age had a better understanding of the relationships between the mind and the brain than younger children, particularly when it comes to mental functions (dream and imagine). However, these relationships were not as well defined for basic functions (see and talk) and scholastic functions (read and count). Children aged 5 to 8 did not perceive the implication of the two entities in cognitive functioning and participants from 10 years to adulthood only recognized the role played by the brain. Our results provide further arguments for the development of neuro-educative school programs based on a better self-awareness of learners in order to help develop young people's metacognitive skills and promote a pedagogical approach that facilitates academic learning.
\end{abstract}

Keywords: Mind-brain conceptions, development, metacognitive skills, neuroeducation.

\section{Introduction}

The notion of "naïve conceptions" corresponds to the way of seeing the world instinctively or naïvely. This term refers to the idea that a naïve conception results from an intuitive knowledge leading to an understanding of natural phenomena, distinguishing from a scientific conception. In this study, we are interested in naïve conceptions about the mind, the brain and their relationships. In cognitive sciences, the common definition claims that the mind refers to the product of brain processes engaged in treatment of intern and extern information. From this point of view, the mind does not exist without the brain and vice versa. Studying naïve mind-brain conceptions is a way to study metacognitive knowledge which is known to promote learning (Wang et al., 1990). While a better understanding of the mind-brain conceptions development could be relevant for education, studies are still scarce. To our knowledge, Johnson and Wellman (1982) conducted the only study that explored children' naïve mind-brain conceptions through the nature and the functions of the mind and the brain in different human activities. Their first study investigated how children consider only the role of the brain in mental acts, sensations, feelings, and voluntary and involuntary behaviours. Children from 5 to 11 years and adults made judgments whether the brain was necessary to these activities. The results indicated that younger children consider the brain necessary for a limited set of activities such as mental acts and to a lesser extent in school activities. From 8/9 years, children progressively showed a better understanding of the brain's role in various activities. However only adults considered the brain as necessary for all activities including involuntary behaviours. In the study 2 , judgments on brain and mind were compared, as well as their material or nonmaterial status. Children between the ages of 6 and 15 years were asked to say where their mind and their brain were, if mind and brain, separately, could be seen or touched, and if one could exist without other. They had also judged if various activities could be performed without the mind or the brain. The authors showed that children aged 6/7 years confused the functions of the mind and the brain, while separating 
them in the head. Afterwards, between 8 and 11 years of age, they became able to understand that the mind and the brain are dependent on each other. Finally, the capacity to materialize the mind in the brain and to understand that the mind produced by the brain only appeared at 14/15 years. Study 3 investigated the ideas about the function of the brain and the study 4 explored their ability to localize the brain inside the head, in 3 to 5 year-old children. At all ages, mental acts were associated with the brain more than sensory-motor activities. Only the younger children were not able to consider that the brain is inside the head. There results were replicated in a contemporary sample with the same design protocol focused on the conceptualization of only the brain (Marshall et al., 2012). Notably, authors also proposed two questions concerning the nature of the mind-brain relation, but unfortunately, they did not report the obtained results in their paper. To our knowledge, no study has focused on these relations by giving children the opportunity to associate (or not associate) the mind and the brain to observe whether children can materialize the mind into the brain. The existent developmental literature emphasized the necessity to revise and extent the comprehension of mind-brain conceptions. Indeed, the heterogeneity in used methodologies and the inconsistency in age groups did not allow us to understand clearly the course of these conceptions until adulthood through different cognitive functions. More recently, Rossi et al. (2015) assessed naïve mind-brain conceptions of children aged 8 years who participated or not in a neuroimaging experiment. This study is interesting because they introduced a new methodology which allows to perceive the real naïve conceptions of participants because it did not only focus on the mind and the brain during the questioning. The employed Mind-Brain questionnaire (MBQ) seems to be a more adapted tool to assess naïve mind-brain conceptions by less influencing the children's responses.

\section{Method}

A total of 34 children aged 5-6 years $(\mathrm{m}=69.5 \pm 3.8$ months; 16 girls $), 34$ children aged $7-8$ years $(\mathrm{m}=95.7 \pm 6.9$ months; 24 girls $), 33$ pre-adolescents aged $10-12$ years $(\mathrm{m}=134.5 \pm 6$ months; 22 girls), 30 adolescents aged $14-16$ years $(m=176.1 \pm 6.1$ months; 17 girls), and 33 adults aged 21 to 35 years $(\mathrm{m}=318.1 \pm 55.4$ months; 15 women $)$ participated in this study. All groups differing in age (all $p s<.001$ ). Adults were recruited according to certain criteria: holding a maximum five years' degree after the baccalaureate and not working in the medical field. Adults' participants and the parents of children and adolescents gave their written consent in order to participate to the study. All participants were tested in accordance with national and international norms governing research on human participants. All participants were recruited in Normandy (France). They hailed from middle class homes and were native speakers of French. None of them reported cognitive impairments or developmental problems. All participants were interviewed individually by using the MBQ. A character, Julie, with her cats was presented to each participant, illustrating by a drawing. We explained that Julie performed different actions with her cats. Then, we introduced the response cards (brain, mind, hand, heart, eye, mouth) that we made sure they are recognized and understood by the participant. For each presented action, the participant was asked "What does Julie need to do this action (e.g., see her cats)?" and was asked to use response cards to answer question, which were placed in front of the participant and randomized between each actions. It was specified that choosing many responses cards was possible and that there was no right or wrong answer. We repeated the method for six cognitive functions presented in random order between each participant (Basic functions: see and talk; Scholastic functions: read and count; Mental functions: dream and imagine). To the extent that our interest is focused on naïve mind-brain conceptions, our interest response cards are "mind" and "brain" and the other response cards are considered as distractors. Then, for each cognitive function, participant's response can be one of the following response category: 1- Mind and Brain response cards; 2- Brain without Mind response cards; 3- Mind without Brain response cards; 4- Neither Mind nor Brain response cards. We conducted repeated-measures analysis of variance for each response category. We used Age as the between factor and Cognitive functions types as the within factor. When the interaction effect was significant, we carried out a posteriori analysis (Dunn-Bonferroni Test). We also performed planned comparisons with a corrected significance threshold for each elementary comparisons at $p=.005$. We supposed that participants should answer more the mind and the brain simultaneously with age to the extent that the acquisition of knowledge increases during development. However, this effect would be different according to cognitive functions.

\section{Results}

Few children aged 5/6 years considered the mind and the brain necessary for all cognitive functions types (Figure 1A). From 7/8 years until adulthood, participants associated these responses more with mental functions than with basic functions and scholastic functions. Furthermore, whatever the age, we observed the same percentage of participants who associated mind and brain responses to basic functions than to scholastic functions. From a developmental point of view, we observed that the number 
of participants who gave mind and brain responses for mental functions increased with age until 10/12 years to reach $80 \%$ of participants at the adulthood. Adults more frequently chose mind and brain responses than 5/6 and 7/8 years children for scholastic functions. The same result was observe between adults and 5-6 years children for basic functions. Then, according to our hypothesis, we observed that participants should answer more the mind and the brain responses simultaneously with age, differently according to cognitive functions. The developmental course seems to include a step at 10 years of age.

Figure 1. Percentages of participants in each response category according to Age and Cognitive functions type. (A) Mind and Brain response category. (B) Mind without Brain response category. (C) Brain without Mind response category. (D) Neither Mind nor Brain response category.
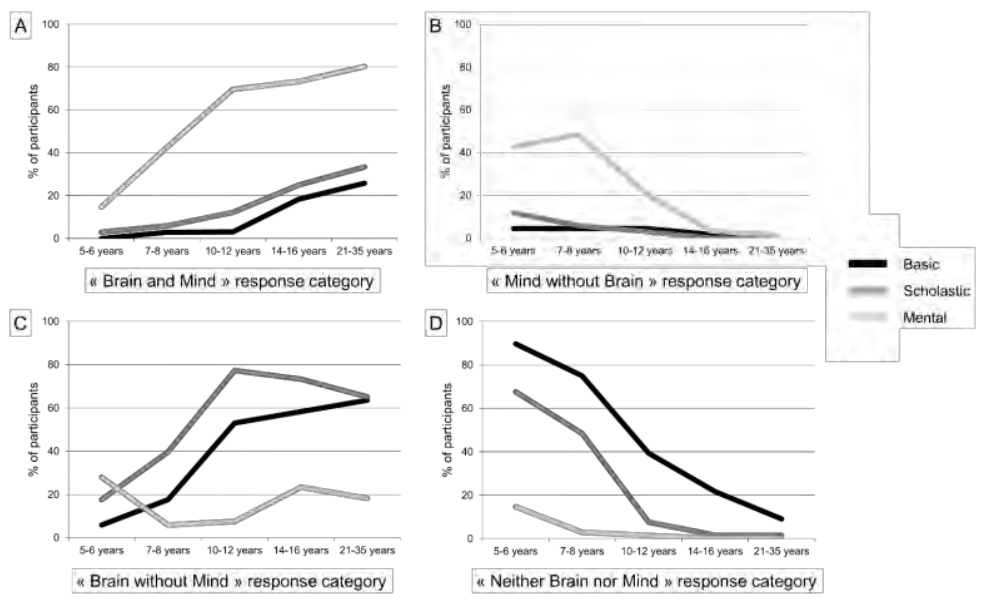

Many children aged 5/6 years and 7/8 years considered only the mind necessary for mental functions contrary to older participants (Figure 1B). No significant developmental difference appeared for basic and scholastic functions for which the mind response was very few given. While we did not observed a significant age effect on the percentage of participants who answered that only the brain was necessary for mental functions, this response was progressively answered for basic functions and scholastic functions (Figure 1C). 5/6 and 7/8 years old children had few considered that the brain was necessary for each function contrary to older participants. Few participants considered that neither the mind nor the brain was necessary for mental functions (Figure 1D). However, this was the case for children before 8 years old for basic functions and scholastic functions contrary to older participants. Finally, we observed a developmental shift in the naïve mind-brain conceptions at 10 years of age (Figure 1).

\section{Conclusion}

Our research reveals an evolution of the naïve mind-brain conceptions during development. The relationships between the mind and the brain are better understanding with age, but only in context of mental functions, not in a context of basic nor scholastic functions. Furthermore, we show that naïve mind-brain conceptions stop to evolve around 10/12 years. Children as adolescents and adults seem to lack knowledge about themselves and their own functioning while this knowledge promote learning and skills emergence. In the neuroeducation context, our results encourage to develop pedagogical programs based on the self-awareness in school learning to develop metacognitive skills and promote academic learning.

\section{References}

Johnson, C. N., \& Wellman, H. M. (1982). Children's developing conceptions of the mind and brain. Child Development, 222-234.

Marshall, P. J., \& Comalli, C. E. (2012). Young Children's Changing Conceptualizations of Brain Function: Implications for Teaching Neuroscience in Early Elementary Settings. Early Education \& Development, 23, 4-23.

Rossi, S., Lanoë, C., Poirel, N., Pineau, A., Houdé, O., \& Lubin, A. (2015). When I Met my brain: Participating in a neuroimaging study influences children's naïve mind-brain conceptions. Trends Neuroscience and Education, 4, 92-97.

Wang, M. C., Haertel, G. D., Walberg, H. J. (1990). What influences learning? A content analysis of review literature. Journal of Educational Research, 84, 30-43. 